\title{
L'INTERTESTUALITA': UN INTERVENTO MACROSTRUTTURALE NELL'AMBITO DEI MECCANISMI DI FUNZIONAMENTO TESTUALE IN DIPENDENZA AL PROBLEMA DELLA TRADUZIONE
}

\section{INTRODUZIONE}

Scopo di questo articolo è l'applicazione descrittiva all'incipit di un testo di narrativa fantascientifica di un segmento macrostrutturale diunoinstrumento d'analisi da noi precedentemente elaborato che si proponeva di sistematizzare e spiegare l'attivazione testuale integrata da alcuni apporti narratologici (come è previsto che il testo sia attivato da chi lo legge), la macroanalisi (come è previsto che il testo funzioni per chi lo legge) e la microanalisi (come è previsto che il testo sia attivato a livello locale da chi lo legge) nel processo della traduzione nel modello a tre fasi a trasferimento indiretto (l'applicazione in questione interessa la prima delle tre fasi del modello).

Con tale strumento ci eravamo posti l'obiettivo di impostare i moduli operativi in modo da poter affrontare la traduzione in termini di configurazione orientata in una prospettiva di trasferimento linguistico, scegliendo un modello preferenziale per tutti i tipi di operazioni linguistiche, tale da possedere delle preferenze per riprodurre mondi testuali su testi di superficie, in cui si mirava a far concorrere la capacità di trasmettere lo stesso genere di esperienza con lo stesso genere di materiale linguistico, accrescendo, riducendo o modificando le componenti testuali solo quando ciò si rendesse necessario per assicurare la più ampia convergenza di esperienze, trattando quindi il testo originale in modo da garantire un ottimo grado di equivalenza, il che presupponeva lo sviluppo prioritario e centrale di un quadro di riferimento che considerasse il testo come una configurazione orientata verso la comunicazione con una dimensione tematica, funzionale e pragmatica.

Questo quadro di riferimento, a sua volta, era stato realizzato (in una prospettiva di economicità e di efficienza, oltre che di perdita ridotta nel trasferimento) attraverso il coinvolgimento di tutti i processi traduttivi nell'analisi testuale: infatti, nella misura in cui le tre dimensioni testuali vengono derivate, attraverso l'analisi testuale, dalla struttura di superficie testuale (dapprima attraverso delle operazioni di definizione macrotestuale e quindi nella segmentazione frastica) la struttura di superficie del testo acquista la dimensione di un insieme strumentale di istruzioni che guida il destinatario alla comprensione del testo in prospettiva al suo più efficace trasferimento. 
Così l'obiettivo di sviluppare la capacità di operare su di un testo (in funzione del trasferimento) attraverso un approccio basato sull'analisi testuale (intesa come strumento per definire la configurazione orientata, individuare le dinamiche di funzionamento del testo e stabilire le modalità operative dei criteri di testualità attraverso il lavoro di segmentazione) aveva trovato quindi ad un tempo modalità di sintesi nell'approccio fondato sull'analisi testuale (nella sua concreta applicazione nelle diverse fasi della traduzione) e modalità di verifica e controllo nel momento della riproduzione del testo in un'altra lingua.

Il modello scelto per l'applicazione dell'analisi testuale (frase estesa) al processo della traduzione era stato quindi individuato (come accennato in apertura) nel modulo a tre fasi a trasferimento indiretto, in cui il traduttore dapprima decodifica il testo macrocontestualmente e microcontestualmente, quindi inizia a coordinare i singoli elementi del testo della lingua di partenza con la lingua d'arrivo, decidendo sulla base di una specifica strategia di trasferimento intralinguistica e, infine, attraverso delle operazioni di sintesi nella lingua di arrivo, produce il testo nella lingua di arrivo: è in prospettiva a questo primo momento di decodificazione macrostrutturale che cercheremo di verificare le modalità di funzionamento, nello sviluppo testuale, dell'ultimo criterio di testualità, l'informatività .

Per la molteplicità e la frequenza dei riferimenti prescrittivi che afferiscono nel paradigma è risultato impossibile segnalare nel corso dell'articolo le relative fonti bibliografiche, in quanto una loro collocazione all'interno dello stesso avrebbe reso estremamente frammentaria la lettura del lavoro. Per la bibliografia utilizzata rimandiamo quindi ai titoli elencati in appendice e per quanto riguarda le indicazioni bibliografiche di cui sopra ai riferimenti presenti nello strumento teorico cui abbiamo accennato precedentemente (HORRAKH L., La macroanalisi e microanalisi testuale nel modello traduttivo in tre fasi a trasferimento indiretto, Edizioni Triestepress, Trieste, 1989).

\section{IL TESTO: "THE THREE STIGMATA OF PALMER ELDRITCH"}

Il testo oggetto d'intervento rappresenta l'incipit (meno di mezza pagina) di un romanzo di fantascienza scritto da P.K. Dick nel 1964, "The Three Stigmata of Palmer Eldritch" (d'ora in poi abbreviato in "Stigmata").

Le dieci righe del testo corrispondono a due paragrafi della narrazione e sono state divise e ridistribuite in diciannove segmenti separati spazialmente per segnalare la scansione narrativa del paragrafo a fini sostanzialmente didascalici, in quanto una simile sgementazione permette di evidenziare immediatamente le proposizioni che compongono la frase e ad un tempo segnala visivamente i rapporti intrattenuti dalle frasi con i segmenti immediatamente adiacenti, a destra e a sinistra. Si tratta, evidentemente, di una segmentazione provvisoria, che non vuole nè dare ragione dell'organizzazione strutturale del testo nè spiegare i meccanismi distributivi, ma 
che si presta paradigmaticamente per una spiegazione progressiva dei diversi moduli d'intervento.

Così

"His head unnaturally aching, Barney Mayerson woke to find himself in an unfamiliar bedroom in an unfamiliar conapt building. Beside him, the covers up to her bare, smooth shoulders, an unfamiliar girl slept on, breathing lighthly through her mouth, her hair a tumble of cotton-like white.

I'll bet I'm late for work, he said to himself, slid from the bed, and tottered to a standing position with eyes shut, keeping himself from being sick. For all he knew he was several hours' drive from his office; perhaps he was not even in the United States. However he was on Earth; the gravity that made him sway way familiar and normal."

è stato segmentato in

1. "His head unnaturally aching"

2. "Barney Mayerson woke"

3. "to find himself in an unfamiliar bedroom"

4. "in an unfamiliar conapt building.

5. "Beside him, the covers up to her bare, smooth shoulders,"

6. "an unfamiliar girl slept on,"

7. "breathing lightly through her mouth,"

8. "her hair a tumble of cotton-like white."

9. "I'll bet I'm late for work,"

10. "he said to himself,"

11. "slid from the bed,"

12. "and tottered to a standing position with eyes shut,"

13. "keeping himself from being sick."

14. "For all he knew"

15. "he was several hours' drive from his office;"

16. "perhaps he was not even in the United States."

17. "However he was on Earth;"

18. "the gravity that made him sway"

19. "was familiar and normal."

\section{L'INTERTESTUALITÀ}

Entrando nel merito dell'intertestualità, dovremmo occuparci di quei fattori che fanno dipendere l'utilizzazione dell'incipit di "Stigmata" dalla conoscenza di uno o più testi accettati in precedenza, verificando quali sono le interdipendenze tra produzione e conoscenze per il processo di mediazione che si mette in atto nell'attivazione delle zone di sapere della nostrà enciclopedia di lettori modello. 


\section{MEDIAZIONE DI ALTRI TIPI TESTUALI}

Innanzittutto le interdipendenze in questione dovranno essere verificate prendendo in considerazione gli altri eventuali tipi testuali che fungono da mediatori nell'utilizzazione dipendente dell'incipit: le dominanze emergenti nell'incipit in questione rimandano necessariamente, per l'utilizzazione di "Stigmata", alla mediazione di superficie, o di largo formato enciclopedico, dei testi narrativi (per come le loro modalità risultano funzionali alla tipologia in questione). In questo senso, l'utilizzazione dell'incipit di "Stigmata" dipende dalla conoscenza della disposizione, in un testo narrativo, in un determinato ordine sequenziale, di azioni e avvenimenti, dell'organizzazione delle relazioni concettuali sotto forma di causa, ragione e prossimità temporale, della frequenza nel testo di superficie di una certa classe di subordinazioni (pre-modificazioni di stato e post-modificazioni di stato nel primo paragrafo, dipendenza lineare nel segmento 13, dipendenza lineare nel segmento 14 , relazioni ellittiche nel macrostato 18-19) e dell'emergenza di un pattern globale come schema (ritrovabile in simili macroproposizioni).

\section{CITAZIONI DI/DA ALTRI TESTI}

Il secondo fattore che fa dipendere l'utilizzazione di "Stigmata" dalla conoscenza di testi pregressi è rappresentato dalla citazione di altri testi: l'elemento di mediazione potrebbe essere rappresentato dalla presenza di "conapt" nel segmento 4 . "Conapt" potrebbe essere un individuo testuale già codificato in altre opere di P.K. Dick, in considerazione della propensione dell'autore a coniare neologismi di tipo descrittivo. E' tuttavia difficile affermare che sia effettivamente così: per farlo bisognerebbe disporre di un formato di enciclopedia che contenga tutte le altre opere di P.K. Dick precedenti la produzione di "Stigmata" (o successive se è diverso l'ordine di lettura).

\section{ALLUSIONI AD ALTRI TESTI}

Il terzo elemento di mediazione è rappresentato dalle allusioni del testo ad altri testi noti. Nell'incipit possiamo individuare due tipi di interdipendenze, a livello superficiale e a livello più profondo. A livello superficiale incontriamo in 17 il fenomeno di ipercodifica "However he was on Earth" che funziona come marca di genere e che quindi fa dipendere l'utilizzazione di "Stigmata" dalla conoscenza di più testi di fantascienza accettati in precedenza (in questo senso il processo di mediazione è sostenuto dalla presenza di altri due individui, "her hair a tumble of cotton-like white" in 8 e dal già visto "conapt building" del segmento 4): tutte le sceneggiature relative agli individui in questione presenti nei relativi macrostati presuppongono un certo formato di una sezione dell'enciclopedia del lettore modello, ove alcune zone di sapere devono necessariamente contenere informazioni relative a particolari individui 
testuali che sono sempre presenti come marche di tipo testuale per sceneggiature massimali nelle fabulae prefabbricate.

A livello più profondo, il processo di mediazione è rappresentato dal perfezionamento isotopico dell'estraneità nei segmenti $14-17$, che funziona come base per stabilire un dato livello di senso autoreferenziale e autocitatorio (autoallusivo) in quanto motivo ricorrente nelle tematiche dei romanzi di P.K. Dick del periodo in cui fu scritto "Stigmata" (anche in questo caso, tuttavia, per poter stabilire una simile interdipendenza, è necessario disporre di un formato settoriale di enciclopedia che contenga la conoscenza dei romanzi di P.K. Dick precedenti la produzione del romanzo in questione).

\section{INTERDIPENDENZE TRA PRODUZIONE E CONOSCENZE TESTUALI}

Il quarto elemento di mediazione è dato dalle interdipendenze tra produzione testuale e conoscenze testuali, in altre parole dai processi di mediazione relativi che permettono, in scala lineare-diacronica, di attivare delle zone di sapere nell'enciclopedia del lettore modello: non risultano essere presenti interdipendenze del quarto tipo in quanto il processo di mediazione relativo è neutralizzato poichè risulta irrilevante il tempo trascorso tra l'uso del testo attuale e l'uso di testi pregressi viste le regole di ipercodifica qui operanti, fatte salve le eventuali citazioni ed allusioni.

\section{MEDIAZIONE TRA PRODUZIONE TESTUALE E CONOSCENZE ATTIVATE ATTRAVERSO FRAME}

Il quinto elemento è infine dato dalla mediazione tra produzione testuale e conoscenze attivate attraverso $i$ frame (qui intesi in senso lato come dispositivi di previsione e di interpretazione da un repertorio di dati conosciuti). A questo livello di macroanalisi ci muoveremo attraverso il controllo dei frame per come questi sono costruiti dal lettore modello a partire dai microstati (concetti) primari e secondari nelle zone di sapere circostanti i relativi macrostati.

Il lavoro in questione si dovrà muovere dai microstati individuabili nel corso dell'analisi conducibile intorno alle modalità operative della coerenza e, basandosi sulle indicazioni ricavabili nel corso dell'attivazione testuale, dovrà ad un tempo verificare se i singoli microstati sono accessibili grazie ad altre mediazioni testuali oppure se essi sono attivati per mezzo del repertorio di dati conosciuti (attraverso sceneggiature comuni, sceneggiature intertestuali, sceneggiature - motivo o sceneggiature convenzionali), secondo quali modalità avviene la mediazione di frame in questione in quanto dispositivo di previsione ed interpretazione dal repertorio di dati conosciuti (enciclopedia), come in questa mediazione sono presenti sei tendenze interattive e come esse sono neutralizzate: entreremo nel merito dei concetti primari e secondari in dipendenza lineare a prescindere dal lavoro di contestualizzazione che 
è svolto nel corso dell'attivazione testuale, in quanto questo tipo di verifica è finalizzato unicamente all'individuazione dei processi di mediazione e non dell'intero processo di contestualizzazione accennato.

Procediamo quindi linearmente attraverso i diversi microstati dell'incipit di "Stigmata". "Head" è un microstato primario contenente un sapere tipico, accessibile attraverso sceneggiature comuni con ridottissima sceneggiatura di frame e probabile assenza di tendenze interattive. "Unnaturally" è un microstato secondario contenente un sapere determinato dal testo attivato attraverso sceneggiature intertestuali con mediazione complessa in cui potrebbero venire erroneamente privilegiate conoscenze ipotizzate nel testo che si accordano con pattern di conoscenze già memorizzate (si tratterà di difendere l'attivazione del microstato da ogni collegamento ad una simile situazione di malessere esperita dal lettore nel corso di esperienze personali). "Aching" è un microstato secondario contenente sapere tipico attivato attraverso sceneggiature comuni con mediazione di frame ridotta in cui potrebbe essere presente una tendenza simile a quella dell'occorrenza precedente e che quindi richiede una difesa. "Barney Mayerson" è un microstato primario contenente sapere determinato dal testo attivabile attraverso sceneggiature-motivo con mediazione ancora ridotta di frame e assenza di tendenze interattive. "Woke" è un microstato primario contenente sapere tipico attivato attraversouna semplice sceneggiatura comune che richiede l'eventuale neutralizzazione della tendenza interattiva precedentemente accennata. "To find himself" è un microstato secondario contenente sapere tipico attivato attraverso una sceneggiatura comune con minima mediazione di frame e assenza di tendenze interattive. "Unfamiliar" è un microstato secondario contenente sapere determinato dal testo accessibile attraverso una duplice sceneggiatura intertestuale e massimale che richiede una notevole mediazione di frame e, quando è co-riferito al microstato (concetto) primario (contenente sapere determinato) "bedroom“, deve essere difeso dall'interferenza di pattern di conoscenze già memorizzate (e l'unfamiliar bedroom" non dovrà aprirsi all'immagine di una qualsiasi stanza d'albergo in cui, nel passato, si è fermato il lettore). Il secondo "unfamiliar" è un microstato secondario contenente sapere determinato dal testo accessibile attraverso sceneggiature intertestuali e massimali che richiede un notevole lavoro di mediazione e che deve essere difeso dalla tendenza a mescolare e confondere elementi diversi delle conoscenze del testo se questi sono strettamente connesi con le conoscenze già memorizzate (il precedente "unfalimiar" che potrebbe rafforzare lo schema di una situazione simile memorizzata). "Conapt" $\mathrm{e}$ "building" sono microstati (concetti) secondari e primari contenenti entrambi un sapere determinato dal testo ed accessibili attraverso sceneggiature comuni e intertestuali con un notevole lavoro di mediazione e devono essere difesi dalla tendenza a modificare le conoscenze presentate dal testo per ottenere un accordo migliore con quelle già memorizzate (per evitare di ottenere una sceneggiatura riduttiva che non tiene conto del necessario lavoro di ipercodifica). "Covers" è un microstato secondario contenente sapere tipico accessibile attraverso una sceneggiatura comune con una minima mediazione di frame in cui non è presente nessuna tendenza interattiva. "Bare" $\mathrm{e}$ "smooth" sono due microstati secondari contenenti sapere tipico accessibili attraverso sceneggiature comuni con una 
certa mediazione di frame, che devono essere difesi dalla prima tendenza interattiva (per evitare un collegamento con simili situazioni vissute dal lettore). "Shoulders" $\mathrm{e}$ un microstato secondario contenente sapere tipico attivabile con una sceneggiatura comune che non richiede mediazioni di frame e non deve essere difeso da tendenze interattive. Il terzo "unfamiliar" è un microstato secondario contenente sapere determinato dal testo, attivabile attraverso una doppia sceneggiatura intertestuale e massimale con notevoli mediazioni di frame, che deve essere difeso dalla tendenza a privilegiare le conoscenze veicolate da "Stigmata" se esse sono collegabili alle entrate principali di un pattern globale applicato come cornice (bisogna fare attenzione alla scelta della corretta isotopia come livello di senso). "Girl" è un microstato primario contenente sapere determinato dal testo accessibile attraverso una doppia sceneggiatura comune e intertestuale con ridotta mediazione di frame che deve essere protetto dalla stessa tendenza interattiva presente in "unfamiliar" (il lettore che abbia optato per delle scelte di vita assolutamente caste non potrà pretendere che Barney Mayerson, che si sveglia accanto ad una ragazza - sconosciuta o meno - abbia fatto lo stesso, per quanto questo lettore ingenuo possa voler forzare il frame verso un mondo poco plausibile). "Slept on" è un microstato secondario contenente sapere tipico accessibile attraverso una sceneggiatura comune con assenza di mediazione di frame ed assenza di tendenze interattive. "Lightly" è un microstato secondario contenente sapere accidentale accessibile attraverso una sceneggiatura comune con minima mediazione di frame che potrebbe dover essere difeso dalla prima tendenza interattiva (è inutile che ill lettore non ingenuo visualizzi la ragazza caratterizzata tra 5 e 7 in base a ricordi di esperienze personali apparentemente simili). "Mouth" è un microstato secondario contenente sapere tipico attivato attraverso una sceneggiatura comune con assenza di mediazione di frame e assenza di tendenze interattive. Le stesse caratteristiche sono presentate dall'individuo "hair". "Tumble" è un microstato secondario contenente sapere determinato dal testo attivabile attraverso sceneggiature comuni con una certa mediazione di frame, e deve essere difeso dalla tendenza a far decadere la conoscenza veicolata dal testo se essa appare casuale rispetto alle conoscenze della realtà (il lettore non dovrà giudicare irrilevante questo particolare). "Cotton-like" e "white" sono due microstati (concetti) secondari contenenti sapere determinato dal testo ed accessibili attraverso sceneggiature intertestuali e massimali con notevoli mediazioni di frame che devono essere difesi dalla tendenza ad indebite aggiunte per ottenere una concordanza con il sapere veicolato dal testo attraverso inferenziazioni.

Nel secondo paragrafo, "bet" e "late" sono due microstati secondari contenenti sapere tipico accessibili attraverso sceneggiature comuni con minima mediazione di frame e assenza di tendenze inerattive. "Work" è un microstato primario contenente sapere determinato dal testo accessibile attraverso una sceneggiatura intertestuale con notevole mediazione di frame che deve essere difeso dalla prima tendenza. "Said to himself" è un microstato secondario contenente sapere tipico accessibile attraverso una sceneggiatura comune con ridotta mediazione di frame e in cui non operano tendenze interattive. "Slid" è un concetto primario contenente sapere tipico attivabile attraverso una sceneggiatura comune con mediazione ridotta che dovrà essere 
eventualmente difeso dalla prima tendenza, così come "bed", microstato secondario contenente sapere determinato dal testo accessibile attraverso una sceneggiatura comune con una notevole mediazione di frame che deve essere ancora difeso dalla prima tendenza a privilegiare le conoscenze veicolate dal testo se esse si acordano con pattern di conoscenze già memorizzate (il fatto che Barney Mayerson si risveglia secondo queste modalità non dovrà essere associato al ricordo di un risveglio doloroso successivo ad una serata alcolica). "Standing", "position", "eyes" e "shut" sono tutti microstati (concetti) secondari contenenti sapere tipico accessibili attraverso sceneggiature comuni con minima mediazione di frame e necessità di difesa dalla tendenza interattiva appena accennata che opera in tutta la rete di relative microcoerenze per l'isotopia relativa. "Keeping from" è un microstato secondario contenente sapere tipico accessibile attraverso una sceneggiatura comune senza mediazione di frame e senza la presenza di tendenze interattive. "Being sick" è un microstato primario contenente sapere determinato dal testo accessibile attraverso una sceneggiatura comune e intertestuale con un certo sforzo di mediazione che dovrà essere ancora difeso dalla prima tendenza interativa. "For all he knew" è un microstato (concetto) primario contenente sapere accidentale accessibile attraverso una sceneggiatura convenzionale con notevole mediazione di frame e deve essere difeso da indebite inferenziazioni. "Several" è un concetto secondario contenente sapere accidentale accessibile attraverso una sceneggiatura comune con una certa mediazione di frame che deve essere eventualmente difeso dalla prima tendenza interattiva. "Hours" è un microstato secondario contenente sapere tipico accessibile attraverso una sceneggiatura comune con assenza di mediazione e assenza di tendenze interattive. "Drive" è un microstato primario contenente sapere accidentale accessibile attraverso sceneggiature comuni e intertestuali che deve essere difeso dalla tendenza a far decadere le conoscenze testuali se queste appaiono casuali rispetto a conoscenze della realtà (trattandosi di un romanzo di fantascienza non si potrà narcotizzare "drive" in quanto l'individuo in questione potrebbe benissimo rimandare ad un referente di "veicolo spaziale" e non di "automobile" con tutto ciò che un'attualizzazione del genere implica per il mondo possibile d'accesso). "Office" è un microstato primario contenente sapere determinato accessibile attraverso sceneggiature comuni e intertestuali con notevoli mediazioni di frame che, ancora una volta, deve essere difeso dalla prima tendenza (non bisognerà, ad esempio, evocare una situazione in cui si è giunti tardi al lavoro). "United States" è un microstato secondario contenente un sapere determinato dal testo accessibile attraverso una sceneggiatura comune e intertestuale che richiede una certa mediazione di frame e che deve essere difeso dalla tendenza a modificare le conoscenze date dal testo per ottenere un accordo migliore con quelle già memorizzate. "Earth" è un microstato primario contenente un sapere tipico, accessibile attraverso una sceneggiatura comune, intertestuale e convenzionale con gran lavoro di mediazione di frame e deve essere difeso dalla tendenza a privilegiare le conoscenze veicolate sin qui dal testo se esse sono collegabili alle entrate principali di un pattern globale applicato come cornice. "Gravity" è un microstato primario contenente sapere determinato dal testo accessibile attraverso una sceneggiatura comune e intertestuale con una certa mediazione di frame in cui sono assenti tendenze interattive. "Familiar" e "normal", infine, sono due microstati primari 
contenenti sapere determinato dal testo accessibili attraverso sceneggiature convenzionali con mediazione media di frame e assenza di tendenze interattive.

\section{BIBLIOGRAFIA}

AA. VV., Dizionario di linguistica, Zanichelli, Bologna, 1979.

AA. VV., Retorica generale, Bompiani, Milano, 1976.

Arcaini E., Introduzione alla linguistica descrittiva, Editrice La Scuola, Brescia, 1980.

Beaugrande de R., Text, Discourse and process, Albex, Norwood, 1980.

Beaugrande de R., Dressler W.U., Introduzione alla linguistica testuale, Il Mulino, Bologna, 1981.

Benard J.P., Horguelin P.A., Pratique de la traduction, Linguatechn Montréal, 1979.

Berruto G., La semantica, Zanichelli, Bologna, 1977.

Boch W., Wort-, Satz-, Textverarbeitung, Kohlhammer, Stuttgart, 1979.

Brown G., Yule G., Analisi del discorso, Il Mulino, Bologna, 1983.

Bussi Parmiggiani E., Una settimana di cronaca, Pitagora, Bologna, 1978.

Cardona G.R., Introduzione all'etnolinguistica, Il Mulino, Bologna, 1976.

Catford J.C., A Linguistic Theory of Translation, Oxford University Press, Oxford, 1965.

Chapman R., Linguistics and Literature, Arnold, London, 1973.

Coulthard M., An Introduction to Discourse Analysis, Longman, London, 1977.

Crystal D., Investigating English Style, Longman, London, 1969.

Dahl O. (ed.), Topic and comment, Contextual Boundness and Focus, Buske, Hamburg, 1974.

Delisle J., De la théorie à la pédagogie: réflections méthodologiques, sta in L'enseignement de l'interprétation et de la traduction, Editions de l'Université de Ottawa, Ottawa, 1981.

Delisle J., L'analyse du discours comme méthode de traduction, Editions de l'Université de Ottawa, Ottawa, 1982.

Dijk van T.A., Facts: the Organization of Propositions in Discourse Comprehension, University of Amsterdam, Amsterdam, 1978.

Dijk van T.A., Macro-structures, Erlbaum, Hillsdale, 1979.

Dijk van T.A., Some Aspects of Text Grammars, Mouton, The Hague, 1972.

Dijk van T.A., Testo e contesto, Il Mulino, Bologna, 1980.

Dressler W., Introduzione alla linguistica del testo, Officina, Roma, 1974.

Dyer D.R., The Measurement of Individual Style, sta in Linguistica, matematica e calcolatori, Olschki, Firenze, 1983.

Eco U., Lector in fabula, Bompiani, Milano, 1979.

Enkvist N.E., Akademi A., Stylistic and textlinguistics, sta in Current Trends in Textlinguistics, ed. by W. W. Dressler, De Gruyter, Berlin-New York, 1977. Gorrman E., Frame Analysis, Harper and Row, New York, 1974. 
Greimas A.J., Semantica strutturale, Rizzoli, Milano, 1969.

Halliday M.A.K., An Introduction to Functional Grammar, Arnold, London, 1985.

Halliday M.A.K., Hasan R., Cohesion in English, Longman, London, 1976.

Hamon P., Semiologia, lessico, leggibilità del testo narrativo, Pratiche, Parma, 1977.

Horrakh L., Didattica della traduzione specializzata di testi pragmatici dalla lingua straniera: un approccio basato sull'analisi testuale, Editre, Trieste, 1987.

Horrakh L., La macroanalisi e microanalisi testuale nel modello traduttivo in tre fasi a trasferimento indiretto, Triestepress, 1989.

Lausberg H., Elementi di retorica, Il Mulino, Bologna, 1969.

Leech G., Semantics, Penguin, Harmondsworth, 1974.

Marchese A., L'officina del racconto, Mondadori, Milano, 1983.

Mistrik J., Exakte Typologie von Texten, Sagner, Munich, 1973.

Newmark P., Approaches to Translation, Prentice Hall, New York, 1981.

Nida A., Componential Analysis of Meaning, Mouton, The Hague, 1975.

Nida A., Vers une théorie partielle du texte, Buske, Hamburg, 1975.

Nida A., Taber C.R., The Theory and Practice of Translation, Brill, Leide, 1969.

Petofi J., Rieser H., Probleme der modelltheoretischen Interpretation von Texten, Buske, Hamburg, 1974.

Petofi J., Reiser H. (eds.), Studies in Text-grammar, Reidel, Dordrecht, 1973.

Prince G., Narratologia, Pratiche, Parma, 1984.

Pugliatti P., Lo sguardo nel racconto, Zanichelli, Bologna, 1985.

Quirk R., Greenbaum S., Leech G., Svartvik J., A Grammar of Contemporary English, Longman, Burnt Mill, 1980.

Reiss K., Texttyp und Uebersetzungsmethode, Scriptor, Kronberg-Taunus, 1976.

Renzi L., Grande grammatica italiana di consultazione, Il Mulino, Bologna, 1988.

Schecker M., Wulderli P. (eds.), Textgrammatik: Beitrage zum Problem der Textualitaet, Niemeyer, Tubingen, 1975.

Schmidt S.J., Teoria del testo, Il Mulino, Bologna, 1982.

Searle J.R., Speech Acts, Cambridge University Press, London, 1969.

Segre C., Avviamento all'analisi del testo letterario, Einaudi, Torino, 1985.

Vigner G., Lire: du texte au sens, Clé International, Paris, 1979.

Vinay J.P., Darbelnet J., Stilistique comparée du français et de l'anglais, Didier, Paris, 1958.

Violi P., Manetti G., L'analisi del discorso, L'Espresso, Roma , 1979.

Wills W., The Science of Translation, Gunter Narr, Tubingen, 1982.

Povzetek

V članku je uporabljen makrostrukturni segment analitičnega instrumenta, ki ga je avtor izdelal že poprej, $z$ namenom, preveriti načine delovanja pripovednega začetka ("incipita") znanstvenofantastičnega besedila. Upoštevajoč dinamiko medbesedilnosti, in še posebej tiste dejavnike, ki postavljajo uporabo pripovednega začetka (incipita) v odvisnosti od poznavanja enega ali več poprej poznanih besedil, se preverjajo soodvisnosti med tvorjenjem besedila in védenjem za proces posredovanja (mediacije), ki nastopi pri aktiviranju védenjskih področij enciklopedije bralca-modela. 